\title{
Life in the atomic age
}

\section{Cell Biology}

by Thomas D. Pollard and William C. Earnshaw

Saunders/Elsevier Science $\cdot 2002$

Hardback, £34.99/\$59.95

Jeremy Thorner

The magnificence of molecules and their roles in the processes involved in cellular life are masterfully explained in this outstanding new textbook. In the prefatory material, the authors admit to having help in writing certain sections from colleagues and other experts in particular fields, and to receiving additional advice from many others. Despite this disclaimer, it is still remarkable that a two-man team was able to produce such a lucid, readily accessible, yet splendidly detailed, up-to-date and comprehensive book. Aside from the inherent difficulties raised by the rapid pace of advance and the vast body of material to be covered, both Pollard and Earnshaw operate highly active research laboratories. That they managed to find the time to craft this book is an amazing feat, and the fact that the final product is excellent is even more awe-inspiring. To put their achievement into further context, consider for a moment some of this book's major 'competitors'. To produce Cell and Molecular Biology, now in its Third Edition (2002), Gerald Karp quit his academic position some time ago and devoted himself exclusively to this endeavor. To generate the encyclopedic texts spearheaded by Bruce Alberts (Molecular Biology of the Cell, 4th Edition, 2002; see book review on E285) and Harvey Lodish (Molecular Cell Biology, 4th Edition, 2000) required, in each case, teams of half-adozen experts as formal co-authors.

What makes this book such a tour de force and a potential classic is that it represents, in essence, a graphically illustrated benchmark for the remarkable progress that has been made in elucidating life processes at the level of 'molecular machines'. Pollard and Earnshaw illustrate fundamental principles with the usual schematic diagrams and colored blobs doing interesting things, which is fine to convey basic concepts, especially to the beginning student. However, they are also utterly unabashed about showing, wherever possible, the three-dimensional structures of the molecules involved at atomic resolu-
More than this, the book

$$
\text { depicts dramatically just }
$$

how far the overall

$$
\text { discipline of cell biology }
$$

has come...

tion. This information is especially beneficial to the advanced student and the practitioner at the bench. Thus, this text achieves the goal of satisfying two distinct constituencies. More than this, the book depicts dramatically just how far the overall discipline of cell biology has come from staring quaintly at the specializations in various cell types at the ultrastructural level using the electron microscope.

The overall organization of the book is excellent and follows a logical flow and progression. It has also benefited from the complementary areas of expertise and different research interests of the two authors. However, perhaps a little sanctimoniously, they state in the introductory material that "this is not a biochemistry book, but..." and, much later on, "this is not a genetics book, but..." However, the entire text highlights over and over just how much the insights we now have about the biology of cells have come from applying the tools of biochemistry, molecular biology and structural biology, and from analyzing function using genetic methods, including those applied to well-established model organisms. Hence, rather than feeling the need to defend the honor of 'cell biology' as a discipline unto itself, I rather wish Pollard and Earnshaw had been more overt, forthright and explicit about celebrating and paying appropriate homage to the current seamless interconnectedness of all the formerly disparate disciplines that now comprise modern molecular and cellular biology .

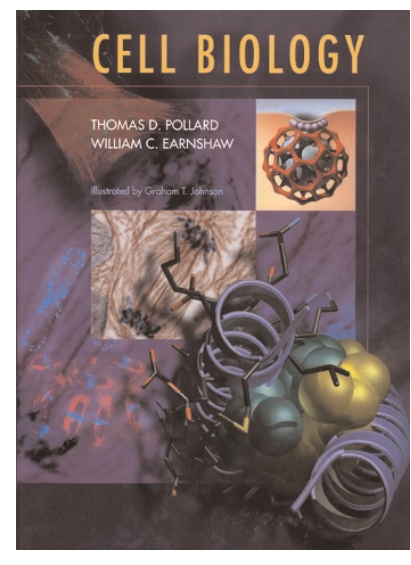

Despite its overall quality, this reviewer has a few other minor quibbles. First, the table of contents is much too telegraphic. After reading the whole, if I wanted to go back to a section I recalled enjoying, I had to thumb through entire chapters to locate what I was looking for. For example, when I went to find again the section on "Transcription Factors and Signal Transduction", I looked first through a major division of the book (chapters 25-29) entitled "Reception and Transduction of Environmental Information." In fact, the section I sought is situated in a major division of the book (chapters 11-16) entitled "Storage and Expression of Genetic Information", admittedly, the next most logical choice. Nonetheless, the section of interest would have been easy to find with a more expanded and detailed table of contents. The second concern is that the book does, regrettably, have errors. It serves no useful purpose to provide a litany of such errors (in fact, the authors urge readers to bring such matters to their attention by contacting them via a web site that they have established). Nevertheless, a few examples, from substantive to typographical, will suffice. It is stated that the $\mathrm{pKa}$ for the $\alpha-\mathrm{NH}_{2}$ group of amino acids is " $\sim 7$ " (page 21), whereas any student in my course knows (hopefully!) that it is 9 or greater. Peter Debye, formulator of many of the fundamental concepts and equations used by biophysicists, gets his name misspelled "Debeye" (page 45). The adenine nucleotideexchange factor for bacterial Hsp70, DnaK, is GrpE, not "GroE", as is mis-stated in multiple places in a figure (Fig. 18-13) and in the accompanying text (page 295). This error no doubt confuses the naive student, who learns in the very next section about chaperonin cages formed from GroEL and GroES.

Jeremy Thorner is in the Department of Molecular and Cell Biology, University of California, Berkeley, CA 94720-3202, USA e-mail: jeremy@socrates.berkeley.edu 